\title{
VARIABILIDADE ESPACIAL DE ATRIBUTOS QUÍMICOS DE UM LATOSSOLO VERMELHO-AMARELO HÚMICO CULTIVADO COM CAFÉ(1)
}

\author{
Samuel de Assis Silva ${ }^{(2)}$, Julião Soares de Souza Lima ${ }^{(3)}$, Alexandre Cândido \\ Xavier $^{(4)} \&$ Mauri Martins Teixeira ${ }^{(5)}$
}

\begin{abstract}
RESUMO
O objetivo do presente estudo foi analisar a variabilidade espacial de atributos químicos de um Latossolo Vermelho-Amarelo húmico. O estudo foi realizado em uma área cultivada com Coffea arabica $\mathrm{L}$. variedade Catuaí. As amostras de solo foram coletadas na profundidade de 0-20 cm, distribuídas em uma malha amostral, com 50 pontos. Os atributos químicos estudados foram: $\mathrm{P}, \mathrm{Na}$ e $\mathrm{S}$ disponíveis, $\mathrm{Ca}$, $\mathrm{Mg}$ e Al trocáveis, $\mathrm{pH}, \mathrm{H}+\mathrm{Al}, \mathrm{SB}, \mathrm{t}, \mathrm{T}, \mathrm{V}, \mathrm{m}, \mathrm{MO}$, ISNa, P-remanescente e micronutrientes ( $\mathrm{Zn}, \mathrm{Fe}, \mathrm{Mn}, \mathrm{Cu}$ e $\mathrm{B}$ ). Os dados foram analisados por meio da análise da estatística descritiva, seguida de uma análise de componentes principais com posterior análise de agrupamento. A geoestatística foi utilizada para verificar a existência e quantificar o grau de dependência espacial dos componentes principais. $\mathrm{O}$ método de análise multivariada com base nos componentes principais forneceu componentes interpretáveis, sendo o primeiro relacionado com a acidez e o segundo com o teor de matéria orgânica do solo. As técnicas de análise multivariada, em associação com a geoestatística, facilitaram a avaliação da variabilidade do solo; os componentes principais 1 e 2 apresentaram dependência espacial moderada, com maior continuidade espacial observada para o componente 1, o que permitiu melhor caracterização da acidez do solo. O solo em estudo apresentou, ao longo da encosta, disponibilidade reduzida de nutrientes, considerável acidez, menores teores de matéria orgânica na porção central da área e valores elevados de $\mathrm{Al}$ trocável na porção superior desta.
\end{abstract}

Termos de indexação: componentes principais, análise de agrupamento, krigagem.

\footnotetext{
(1) Recebido para publicação em julho de 2008 e aprovado em outubro de 2009.

(2) Mestrando em Produção Vegetal, Universidade Federal do Espírito Santo - UFES. Caixa Postal 16, CEP 29500-000 Alegre (ES). Bolsista CAPES. E-mail: samuel-assis@hotmail.com

${ }^{(3)}$ Professor Associado, Departamento de Engenharia Rural, UFES. E-mail: limajss@yahoo.com.br

(4) Professor Adjunto, Departamento de Engenharia Rural, UFES. E-mail: xavier@cca.ufes.br

(5) Professor Associado, Departamento de Engenharia Agrícola, Universidade Federal de Viçosa - UFV. Av. Peter Henry Rolfs s/n, CEP 36570-000 Viçosa (MG). E-mail: mauri@ufv.br
} 


\title{
SUMMARY: SPATIAL VARIABILITY OF CHEMICAL ATTRIBUTES OF AN OXISOL UNDER COFFEE CULTIVATION
}

\begin{abstract}
The objective of this study was to analyze the spatial variability of chemical attributes of an Oxisol. The experiment was conducted with Coffea arabica L., variety Catuai, at a depth of 0-0.2 $\mathrm{m}$ in a grid area totaling 50 points. The studied chemical attributes were: $P, K, C a, M g$, $\mathrm{Na}, \mathrm{S}, \mathrm{Al}, \mathrm{pH}, \mathrm{H}+\mathrm{Al}, \mathrm{SB}, t, \mathrm{~T}, \mathrm{~V}, \mathrm{~m}, \mathrm{MO}$, ISNa, equilibrium Pand micronutrients ( $\mathrm{Zn}, \mathrm{Fe}, \mathrm{Mn}$, $\mathrm{Cu}$, and $\mathrm{B})$. The data were analyzed by descriptive statistical analysis, followed by principal component with subsequent cluster analysis. The existence of spatial dependence was verified and the degree quantified using the principal components and geostatistics. The method of multivariate analysis based on principal components provided interpretable components; the first were correlated with acidity and the second with soil organic matter. Techniques of multivariate analysis, in combination with geostatistics, facilitated the evaluation of soil variability. The principal components 1 and 2 indicated moderate spatial dependence, with greater spatial continuity in component 1, which allowed a better characterization of soil acidity. Reduced availability of soil nutrients and considerable acidity were observed along the slope, lower levels of organic matter in the center of the area and high Al contents in the upper part.
\end{abstract}

Index terms: Principal components, cluster analysis, kriging.

\section{INTRODUÇÃO}

Os diversos atributos químicos do solo comportamse de forma bastante diferenciada ao longo das áreas cultivadas, devido, entre outros fatores, às alterações provocadas pelo manejo agrícola. Silva \& Chaves (2001) comentaram que os atributos químicos apresentam maior variação que os físicos em uma área cultivada e, devido a esse fato, o uso de amostragens aleatórias, empregando a média para caracterizar determinado atributo no solo, pode resultar em avaliações inexatas.

O conhecimento da variação de atributos químicos é importante para o levantamento e manejo do solo, planejamento de esquemas de amostragem e gerenciamento de práticas agrícolas. Por isso, antes de buscar qualquer relação desses atributos com a cultura, é importante avaliar a extensão e a intensidade da dependência espacial da sua variação, isoladamente ou em conjunto com outras variáveis (Gandah et al., 2000).

Para analisar a estrutura da fertilidade dos solos, é necessária a avaliação dos diversos nutrientes que respondem por ela, o que, muitas vezes, ao ser realizado por meio de métodos estatísticos univariados, eleva a dimensão do problema, além de onerar o processo. Uma opção de análise para esses tipos de dados é o uso de análise multivariada, mais especificamente a análise de componentes principais. E um tipo de análise que tem como vantagens a redução dimensional do problema e a facilidade de interpretação (Bueno, 2001).

O objetivo da análise de componentes principais (ACP) é explicar a estrutura da variância e da covariância de um vetor aleatório, composto de pvariáveis aleatórias, por meio da construção de combinações lineares das variáveis originais. Essa combinação é chamada de componentes principais, os quais não são correlacionados entre si (Mingoti, 2007).

A ACP é um método de ordenação com grande tradição em diversos estudos. Como exemplo de seu uso com variáveis de solo, podem-se citar os estudos com padrões regionais de diferenciação de solos na Amazônia colombiana (Lips \& Duivenvoorden, 1996); o regime de nutrientes em diferentes solos florestais de uma costa montanhosa do Canadá (Splechtna \& Klinka, 2001); as relações entre propriedades abióticas e bióticas do solo durante períodos de pousio no Senegal (Manlay et al., 2000); e as relações entre atributos climáticos, pedológicos e vegetacionais com a ocorrência da macaúba em Minas Gerais (Motta et al., 2002).

Outra técnica muito empregada é a análise de agrupamento, que tem o objetivo de agrupar parcelas de acordo com o grau de similaridade. Basicamente, as técnicas de classificação buscam arranjar um conjunto de observações - cada um com múltiplas variáveis - de modo a agrupar as mais similares e separar as mais dissimilares, formando diferentes grupos. A medida da similaridade entre observações é determinada pela análise simultânea de todas as variáveis em relação às demais. Essas observações podem ser quantitativas ou qualitativas (Gurgel, 2003).

O objetivo do presente estudo foi analisar a variabilidade espacial de atributos químicos de um Latossolo Vermelho-Amarelo húmico cultivado com café, utilizando técnicas de análise estatística multivariada e geoestatística. 


\section{MATERIAL E MÉTODOS}

O estudo foi realizado em uma área cultivada com Coffea arabica L. variedade Catuaí, no espaçamento de $1,0 \times 0,5 \mathrm{~m}$, com declividade acentuada $\left(30 \mathrm{~cm} \mathrm{~m}^{-1}\right)$, localizada na fazenda Jaguaraí, no município de Reduto, região leste do Estado de Minas Gerais, a $20^{\circ} 45^{\prime} 45,4$ " de latitude $\mathrm{Se} 41^{\circ} 32^{\prime} 9,75$ " de longitude W. O solo da parcela experimental foi classificado como Latossolo Vermelho-Amarelo húmico com horizonte A bastante espesso e rico em matéria orgânica, conforme Embrapa (2006).

Para o mapeamento da fertilidade do solo construiu-se uma malha de amostragem, totalizando 50 pontos georreferenciados (Figura 1), nos quais as amostras de solo foram coletadas na projeção das copas de três plantas, na profundidade de $0-20 \mathrm{~cm}$, com auxílio de uma sonda inoxidável de $5 \mathrm{~cm}$ de diâmetro interno. As três amostras de solo de cada ponto foram homogeneizadas, formando as denominadas células de amostragens.

Os atributos químicos estudados foram: $\mathrm{P}, \mathrm{K}, \mathrm{S}$ e $\mathrm{Na}$ disponíveis, acidez ativa ( $\mathrm{pH}$ em água), acidez trocável $\left(\mathrm{Al}^{3+}\right)$ e acidez potencial $(\mathrm{H}+\mathrm{Al})$, soma das bases trocáveis (SB), capacidade efetiva de troca de cátions [CTC(t)], capacidade de troca de cátions a $\mathrm{pH}$ 7 [CTC(T)], índice de saturação por bases (V), índice de saturação por $\mathrm{Al}^{+3}(\mathrm{~m})$, teor de matéria orgânica (MO), índice de saturação por sódio (ISNa), Premanescente e teor de micronutrientes disponíveis (Zn, Fe, Mn, Cu e B).

Os métodos de análise laboratorial, bem como os extratores, para a determinação dos atributos do solo seguiram as recomendações descritas no manual de métodos de análises de solo da Embrapa (1997).

Os valores encontrados foram analisados por meio das medidas de posição e dispersão na análise da estatística descritiva e exploratória, bem como aplicouse o teste de Shapiro-Wilk's $(\mathrm{p}<0,05)$ para testar a

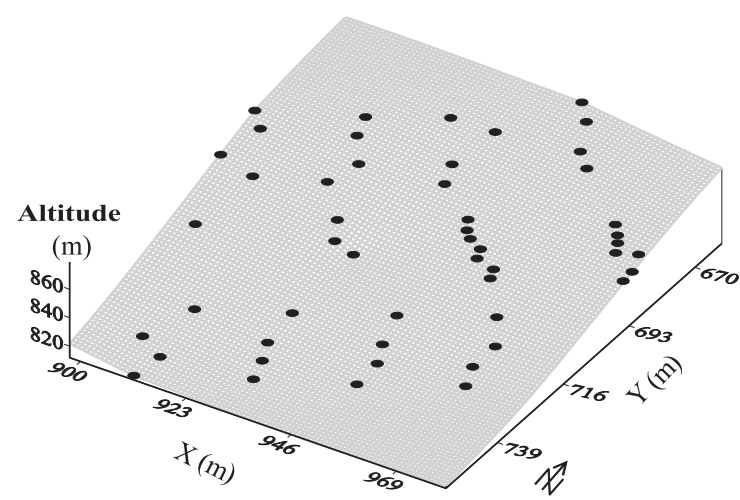

Figura 1. Modelo digital de elevação (MDE) da área em estudo, com a distribuição dos pontos amostrais. normalidade do conjunto de dados, utilizando-se o software Statistica 6.0 (Statsoft, 2001).

A análise de componentes principais (ACP) foi realizada com base na matriz de correlação existente entre as componentes e os atributos reais, de forma a identificar novas variáveis que explicam a maior parte da variabilidade, gerando-se novos valores para cada ponto amostral correspondente aos componentes principais.

Foi construído o círculo de autovetores das variáveis, representados pelos eixos $x$ e $y$ (respectivamente, primeiro e segundo componentes). Em uma matriz original de dados, cada sítio tem um valor para cada atributo. Na ACP, cada sítio tem um valor para cada componente. Esses componentes podem ser vistos como "supervariáveis", construídas pela combinação da correlação entre as variáveis, e são extraídos em ordem decrescente de importância, em termos de sua contribuição para a variação total dos dados. Os autovetores são valores que representam o peso de cada variável em cada componente (eixos) e funcionam como coeficientes de correlação, que variam de -1 até +1. As variáveis com elevado autovetor no primeiro eixo tendem a ter autovetor inferior no segundo eixo. Os autovalores são valores que representam a contribuição relativa de cada componente para explanar a variação total dos dados, existindo um para cada componente. Matematicamente, o processo de extrair mais eixos pode ir até o número de eixos ou componentes igualar-se ao número de variáveis; contudo, os eixos posteriores vão contribuir cada vez menos para explicar os dados. Na maioria dos estudos, usam-se apenas os dois primeiros eixos, considerados suficientes para explicar os dados e pela facilidade de interpretação de um gráfico bidimensional.

Uma análise de agrupamento ("cluster analysis"), segundo o método Ward, foi realizada com o intuito de classificar os atributos químicos do solo e os componentes principais em grupos homogêneos. Essa análise multivariada não leva em consideração a distribuição dos dados, sendo o agrupamento feito com base nas medidas de similaridade entre os indivíduos (Mingoti, 2007).

A seleção do número de componentes principais foi baseada no critério de análise da qualidade de aproximação da matriz de correlações, utilizando-se os componentes associados a autovalores superiores a 1 (Mingoti, 2007). No caso da correlação dos componentes com os atributos químicos do solo, consideraram-se significativos os valores superiores a 0,7 , conforme Zwick \& Velicer (1986).

A geoestatística foi utilizada para verificar a existência e, nesse caso, quantificar o grau de dependência espacial dos dados gerados por meio da aplicação da ACP, o que foi feito a partir do ajuste de funções teóricas aos modelos de semivariogramas experimentais, com base na pressuposição de estacionaridade da hipótese intrínseca e conforme a equação: 


$$
\gamma^{*}(h)=\frac{1}{2 N(h)} \sum_{i=1}^{N(h)}\left[z\left(x_{i}\right)-z\left(x_{i}+h\right)\right]^{2}
$$

em que $\mathrm{N}(\mathrm{h})$ é o número de pares experimentais de observações $\mathrm{Z}\left(\mathrm{x}_{\mathrm{i}}\right), \mathrm{Z}\left(\mathrm{x}_{\mathrm{i}}+\mathrm{h}\right)$, separados por um vetor $\mathrm{h}$ (Vieira et al., 1983; Vieira, 2000).

No ajuste dos modelos teóricos aos semivariogramas experimentais, determinaram-se, com o software GS+ (Robertson, 1998), os coeficientes efeito pepita $\left(\mathrm{C}_{0}\right)$, patamar $\left(\mathrm{C}_{0}+\mathrm{C}_{1}\right)$, variância estrutural $\left(\mathrm{C}_{1}\right)$ e alcance (a). Esse software aplica o método dos mínimos quadrados e adota como critérios para a seleção o maior valor de $\mathrm{R}^{2}$ (coeficiente de determinação), a menor SQR (soma de quadrados dos resíduos) e o maior valor do coeficiente de correlação obtido pelo método de validação cruzada (Guimarães, 2000).

Para análise do índice de dependência espacial (IDE), foi utilizada a relação $\mathrm{C}_{0} /\left(\mathrm{C}_{0}+\mathrm{C}_{1}\right)$ e os intervalos propostos por Cambardela et al. (1994), que consideraram a dependência espacial fraca (IDE $>75 \%$ ), moderada (25\% $\leq$ IDE $\leq 75 \%$ ) e forte (IDE $<25 \%$ ).

Comprovada a dependência espacial, utilizou-se o método de interpolação de krigagem ordinária para estimar valores em locais não medidos (Vieira, 2000).

\section{RESULTADOS E DISCUSSÃO}

No quadro 1 é apresentada a análise exploratória dos valores encontrados para os atributos em estudo. As medidas de tendência central (média e mediana) foram semelhantes para mais de $85 \%$ dos atributos, indicando uma distribuição onde os dados apresentaram reduzido afastamento em relação a um valor central - fato confirmado pelos valores do teste de normalidade de Shapiro-Wilk's a $5 \%$.

Os valores médios dos atributos químicos do solo foram classificados de acordo com Ribeiro et al. (1999) para o Estado de Minas Gerais em: baixos para P, Ca, $\mathrm{Mg}, \mathrm{SB}, \mathrm{V}$, m e Cu; médios para $\mathrm{pH}, \mathrm{K}, \mathrm{Al}$, t, T, MO, $\mathrm{Zn}, \mathrm{Mn}$; e altos para $\mathrm{H}+\mathrm{Al}$, Fe e B.

O coeficiente de assimetria é utilizado para caracterizar como e quanto a distribuição de frequências se afasta da simetria: se $\mathrm{Cs}>0$, tem-se a distribuição assimétrica à direita; se $\mathrm{Cs}_{\mathrm{s}}<0$, a distribuição é assimétrica à esquerda; e se $\mathrm{Cs}_{\mathrm{s}}=0$, a distribuição é simétrica (Guimarães, 2004). Valores negativos de assimetria, conforme observado para ISNa, MO, P-rem e S, indicam distribuição assimétrica à esquerda, enquanto os valores positivos de assimetria para os demais atributos indicam distribuição assimétrica à direita.

Quadro 1. Estatística descritiva dos atributos químicos do solo

\begin{tabular}{|c|c|c|c|c|c|c|c|c|c|}
\hline \multirow{2}{*}{ Variável } & \multicolumn{8}{|c|}{ Estatística } & \multirow[b]{2}{*}{$\mathbf{w}$} \\
\hline & Média & Med. & Mín. & Máx. & $\mathbf{s}$ & CV (\%) & Cs & Ck & \\
\hline $\mathrm{pH}$ & 5,10 & 5,08 & 4,62 & 5,62 & 0,24 & 4,79 & 0,10 & $-0,48$ & $\mathrm{~N}$ \\
\hline $\mathrm{P}^{(1)}$ & 3,64 & 3,40 & 0,90 & 8,20 & 1,86 & 51,15 & 0,49 & $-0,07$ & $\mathrm{~N}$ \\
\hline $\mathrm{K}^{(1)}$ & 89,91 & 90,00 & 24,00 & 186,00 & 38,17 & 42,45 & 0,42 & $-0,26$ & $\mathrm{~N}$ \\
\hline $\mathrm{Na}$ & 3,97 & 3,80 & 2,90 & 5,70 & 0,70 & 17,68 & 0,52 & 0,23 & * \\
\hline $\mathrm{Ca}^{(2)}$ & 1,17 & 1,09 & 0,30 & 2,23 & 0,45 & 38,15 & 0,52 & $-0,17$ & $\mathrm{~N}$ \\
\hline $\mathrm{Mg}^{(2)}$ & 0,40 & 0,37 & 0,09 & 0,82 & 0,17 & 42,90 & 0,46 & $-0,22$ & $\mathrm{~N}$ \\
\hline $\mathrm{Al}^{(2)}$ & 0,54 & 0,50 & 0,00 & 1,30 & 0,32 & 58,95 & 0,08 & $-0,51$ & $\mathrm{~N}$ \\
\hline $\mathrm{H}+\mathrm{Al}$ & 5,38 & 5,40 & 3,65 & 7,43 & 0,80 & 14,84 & 0,23 & 0,05 & $\mathrm{~N}$ \\
\hline $\mathrm{SB}$ & 1,78 & 1,68 & 0,57 & 3,13 & 0,62 & 35,03 & 0,36 & $-0,36$ & $\mathrm{~N}$ \\
\hline $\mathrm{t}$ & 2,41 & 2,35 & 1,37 & 3,77 & 0,51 & 21,35 & 0,44 & 0,02 & $\mathrm{~N}$ \\
\hline $\mathrm{T}$ & 7,18 & 7,18 & 6,00 & 8,74 & 0,67 & 9,39 & 0,22 & $-0,10$ & $\mathrm{~N}$ \\
\hline $\mathrm{V}$ & 24,88 & 23,40 & 8,80 & 46,40 & 8,44 & 33,91 & 0,45 & $-0,09$ & $\mathrm{~N}$ \\
\hline $\mathrm{m}$ & 23,88 & 23,40 & 0,00 & 58,40 & 15,12 & 63,30 & 0,15 & $-0,75$ & $\mathrm{~N}$ \\
\hline $\mathrm{ISNa}$ & 0,74 & 0,75 & 0,39 & 1,12 & 0,16 & 21,23 & $-0,08$ & $-0,22$ & $\mathrm{~N}$ \\
\hline MO & 3,73 & 3,75 & 2,95 & 4,37 & 0,33 & 8,83 & $-0,37$ & $-0,06$ & $\mathrm{~N}$ \\
\hline P-rem & 20,32 & 20,60 & 11,30 & 29,00 & 4,19 & 20,60 & $-0,24$ & $-0,53$ & $\mathrm{~N}$ \\
\hline $\mathrm{Zn}^{(1)}$ & 2,25 & 2,30 & 0,50 & 4,90 & 1,04 & 46,40 & 0,60 & 0,27 & $\mathrm{~N}$ \\
\hline $\mathrm{Fe}^{(1)}$ & 96,40 & 89,60 & 54,00 & 158,20 & 28,61 & 29,67 & 0,57 & $-0,69$ & * \\
\hline $\mathrm{Mn}^{(1)}$ & 7,30 & 7,25 & 2,70 & 11,60 & 2,15 & 29,50 & 0,08 & $-0,54$ & $\mathrm{~N}$ \\
\hline $\mathrm{Cu}^{(1)}$ & 0,30 & 0,30 & 0,10 & 0,60 & 0,11 & 38,44 & 0,56 & 0,01 & * \\
\hline $\mathrm{B}^{(1)}$ & 1,13 & 1,11 & 0,72 & 1,67 & 0,20 & 17,74 & 0,47 & 0,09 & $\mathrm{~N}$ \\
\hline $\mathrm{S}^{(1)}$ & 11,96 & 12,30 & 2,90 & 19,60 & 3,43 & 28,69 & $-0,38$ & 0,39 & $\mathrm{~N}$ \\
\hline
\end{tabular}

(1) Disponíveis. ${ }^{(2)}$ Trocáveis; Med.: mediana; Máx.: máximo; Mín.: mínimo; s: desvio-padrão; CV( \%): coeficiente de variação; C assimetria; $\mathrm{C}_{\mathrm{k}}$ : curtose; $\mathrm{pH}$ em $\mathrm{H}_{2} \mathrm{O} ; \mathrm{P}, \mathrm{K}, \mathrm{Na}, \mathrm{Zn}, \mathrm{Fe}, \mathrm{Mn}, \mathrm{Cu}, \mathrm{B}$ e S em mg dm ${ }^{-3}$; Ca, Mg, Al, H + Al, SB, t, T em cmol dm ${ }^{-3}$; V, m e ISNa em \%; MO em dag kg-1; P-rem em $\mathrm{mg} \mathrm{L}^{-1}$; *: distribuição não normal pelo teste de Shapiro-Wilk's a 5 \%; N distribuição normal pelo teste de Shapiro-Wilk's a $5 \%$. 
Quanto ao coeficiente de curtose, que mostra a dispersão (achatamento) da distribuição em relação a um padrão, geralmente a curva normal, em que: se $\mathrm{Ck}=0$ a distribuição é mesocúrtica, se $\mathrm{Ck}<0$ platicúrtica e se $\mathrm{Ck}>0$ leptocúrtica, a maior parte dos dados apresentou distribuição platicúrtica, porém com achatamento reduzido da curva de distribuição normal, uma vez que esses valores encontraram-se próximos de zero.

Analisando o coeficiente de variação (CV), observou-se que, segundo a classificação proposta por Warrick \& Nielsen (1980), considerando como valores baixos o $\mathrm{CV}<12 \%$, médios para $12 \%<\mathrm{CV}<60 \%$ e altos para $\mathrm{CV}>60 \%$; com exceção de m (alto), pH, T e MO (baixo), os demais atributos encontram-se no intervalo de 12 a $60 \%$, sendo, portanto, considerados de média variação.

Na análise de componentes principais, foram extraídos cinco componentes, que, de forma acumulada, explicam 78,70 \% da variabilidade total dos dados (Quadro 2). Os demais contribuíram de forma irrisória e, portanto, não foram considerados na análise.

O primeiro componente principal, aqui denominado acidez do solo, apresentou isoladamente 42,00\% da variabilidade total dos dados e correlacionou-se com sete dos 22 atributos químicos analisados (Quadro 3). Entre os atributos que se correlacionaram de maneira elevada e negativa (correlação maior que 0,70) com o primeiro componente estão: $\mathrm{pH}, \mathrm{K}, \mathrm{Ca}, \mathrm{Mg}, \mathrm{SB}, \mathrm{t}$ e V. Com isso, e como se observa na figura 2, esse componente sofreu influência negativa da fertilidade do solo, uma vez que esses atributos representam a porção fértil do solo e estão correlacionados de forma negativa com esse componente.

Diante desses resultados, pode-se dizer que o solo em questão apresentou, de modo geral, baixa fertilidade, uma vez que valores de correlação negativos, associados a valores reais aquém do recomendado para tais atributos (Quadro 1), indicam que estes se encontram em níveis não satisfatórios para o bom desenvolvimento das plantas.

Observando os atributos com correlações elevadas e positivas com o primeiro componente, foram encontrados $\mathrm{Al}$ e m. Assim, quando os teores de $\mathrm{Al}$ se elevam,

Quadro 2. Resumo dos componentes principais da análise multivariada

\begin{tabular}{cccc}
\hline \multirow{2}{*}{ Componente } & Autovalor & \multicolumn{2}{c}{ Percentagem } \\
\cline { 3 - 4 } & & Variância & Acumulada \\
\hline 1 & 9,24 & 42,00 & 42,00 \\
2 & 2,97 & 13,51 & 55,51 \\
3 & 2,20 & 10,04 & 65,55 \\
4 & 1,78 & 8,10 & 73,65 \\
5 & 1,10 & 5,05 & 78,70 \\
\hline
\end{tabular}

Quadro 3. Correlação entre variáveis originais e componentes principais dos atributos químicos do solo

\begin{tabular}{lrrrrr}
\hline Variável & $\mathbf{C 1}$ & $\mathbf{C 2}$ & $\mathbf{C 3}$ & $\mathbf{C 4}$ & $\mathbf{C 5}$ \\
\hline $\mathrm{pH}$ & $-0,93$ & $-0,19$ & $-0,09$ & 0,00 & $-0,11$ \\
$\mathrm{P}^{(1)}$ & $-0,18$ & 0,38 & $-0,18$ & $-0,14$ & 0,46 \\
$\mathrm{~K}^{(1)}$ & $-0,80$ & 0,22 & 0,00 & $-0,11$ & $-0,24$ \\
$\mathrm{Na}^{(1)}$ & $-0,37$ & 0,05 & $-0,84$ & 0,15 & $-0,09$ \\
$\mathrm{Ca}^{(2)}$ & $-0,97$ & 0,07 & 0,08 & 0,05 & 0,05 \\
$\mathrm{Mg}^{(2)}$ & $-0,93$ & $-0,05$ & 0,01 & 0,06 & $-0,01$ \\
$\mathrm{Al}{ }^{(2)}$ & 0,79 & 0,47 & 0,15 & $-0,04$ & $-0,15$ \\
$\mathrm{H}+\mathrm{Al}$ & 0,56 & 0,77 & 0,06 & 0,09 & 0,14 \\
$\mathrm{SB}$ & $-0,99$ & 0,08 & 0,04 & 0,02 & $-0,02$ \\
$\mathrm{t}$ & $-0,91$ & 0,31 & 0,13 & 0,01 & $-0,10$ \\
$\mathrm{~T}$ & $-0,52$ & 0,79 & 0,10 & 0,11 & 0,11 \\
$\mathrm{~V}$ & $-0,97$ & $-0,19$ & 0,02 & 0,00 & 0,01 \\
$\mathrm{~m}$ & 0,86 & 0,32 & 0,09 & $-0,04$ & $-0,22$ \\
$\mathrm{ISNa}$ & 0,22 & $-0,14$ & $-0,91$ & 0,14 & $-0,03$ \\
$\mathrm{MO}$ & $-0,32$ & 0,79 & $-0,03$ & 0,31 & 0,00 \\
$\mathrm{P}-\mathrm{rem}$ & $-0,61$ & $-0,19$ & 0,39 & $-0,06$ & $-0,03$ \\
$\mathrm{Zn}^{(1)}$ & $-0,19$ & 0,27 & $-0,1$ & $-0,79$ & 0,04 \\
$\mathrm{Fe}{ }^{(1)}$ & 0,30 & $-0,01$ & 0,15 & 0,07 & $-0,73$ \\
$\mathrm{Mn}^{(1)}$ & $-0,11$ & 0,52 & $-0,31$ & $-0,25$ & $-0,29$ \\
$\mathrm{Cu}(1)$ & $-0,11$ & $-0,01$ & $-0,10$ & $-0,92$ & $-0,09$ \\
$\mathrm{~B}^{(1)}$ & $-0,47$ & 0,11 & 0,37 & 0,02 & $-0,13$ \\
$\mathrm{~S}^{(1)}$ & 0,42 & $-0,10$ & 0,35 & $-0,24$ & 0,23 \\
\hline $\mathrm{Disp}$ & & & & &
\end{tabular}

(1) Disponíveis. ${ }^{(2)}$ Trocáveis.

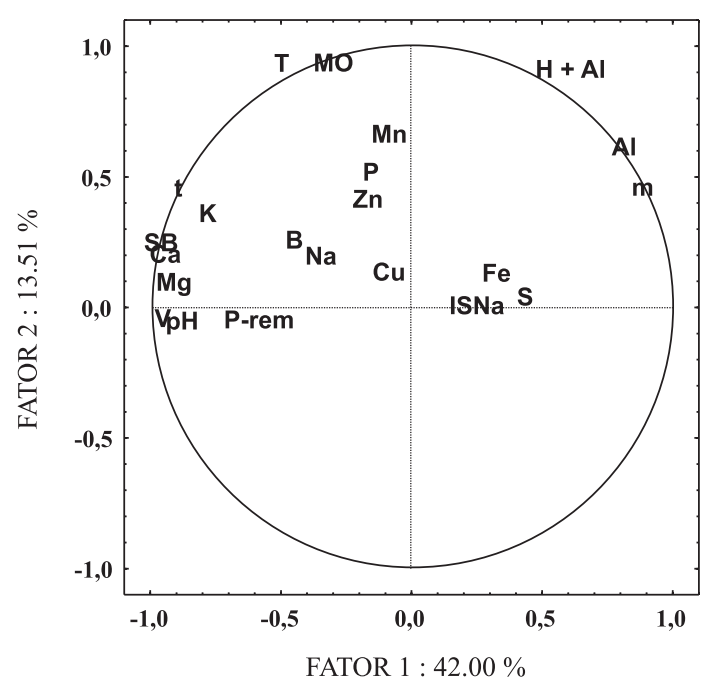

Figura 2. Círculo das correlações do plano formado pelos componentes principais 1 e 2 .

indicam redução na fertilidade do solo, caracterizada pela baixa disponibilidade dos demais nutrientes na área em estudo.

No segundo componente principal, que explicou $13,51 \%$ da variabilidade total dos dados, aqui denominado fator orgânico, apenas os atributos $\mathrm{H}+\mathrm{Al}$, T e MO apresentaram correlação significativa. Assim, pode-se dizer que esse componente está relacionado à 
parte orgânica do solo, visto que sua correlação com a MO e a CTC (T) é elevada e esta, por sua vez, sofre influência da primeira.

Valor de correlação significativo e positivo com o teor de MO já era esperado, em razão da classe de solo sobre a qual o estudo foi desenvolvido. De acordo com Ker (1997), a distrofia, característica marcante desses solos, aliada a temperaturas amenas, comum nas suas regiões de ocorrência, inibem a atividade microbiana, favorecendo o acúmulo de matéria orgânica, principalmente no horizonte $\mathrm{A}$, que pode apresentar profundidade superior a $1 \mathrm{~m}$.

A técnica de análise de agrupamento permitiu complementar, de maneira eficiente, os resultados obtidos com o uso da análise de componentes principais (Figura 3). Com os 22 atributos originais, foi utilizado o método Ward com a medida de similaridade dada pela distância euclidiana, com o objetivo de agrupálos, definindo a distância de corte igual a 400, formando assim dois grupos homogêneos.

No agrupamento realizado com os cinco componentes principais (Figura 4), o dendrograma mostra a formação de dois grupos de parcelas homogêneas com base no corte a uma distância igual a 20.

A figura 5 representa a distribuição dos pontos amostrais, explicitando os dois grupos formados na análise de agrupamento com os dados reais (5A) e com os cinco componentes principais (5B).

Observou-se similaridade entre o conjunto real de atributos avaliados e os cinco componentes principais (Figura 5). Essa similaridade atesta a utilização da análise que reduziu as variáveis em um menor número, facilitando a interpretação.

Comparativamente, a análise de agrupamentos por meio dos cinco componentes principais reduz a complexidade da interpretação dos atributos químicos

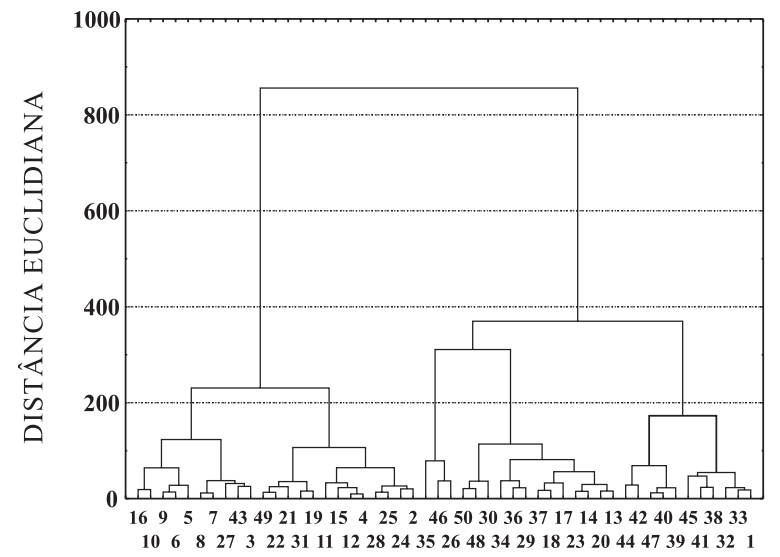

Figura 3. Dendrograma ilustrativo resultante da análise de agrupamento dos atributos químicos do solo.

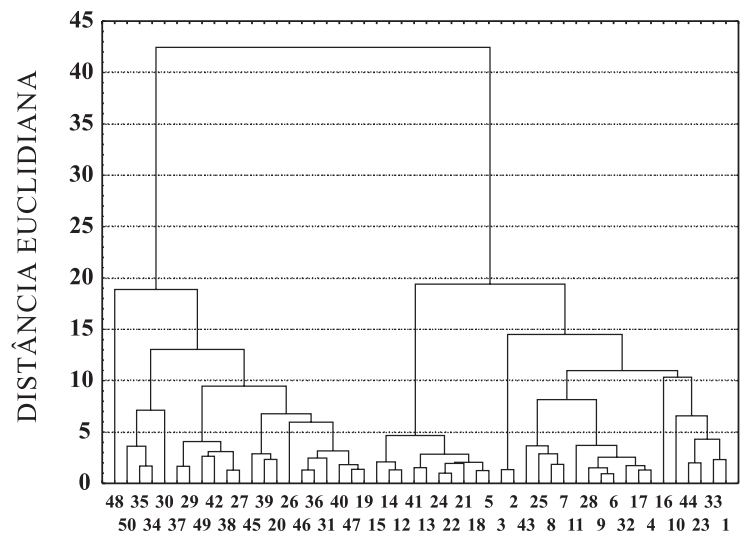

Figura 4. Dendrograma ilustrativo resultante da análise de agrupamento dos nove primeiros componentes principais.

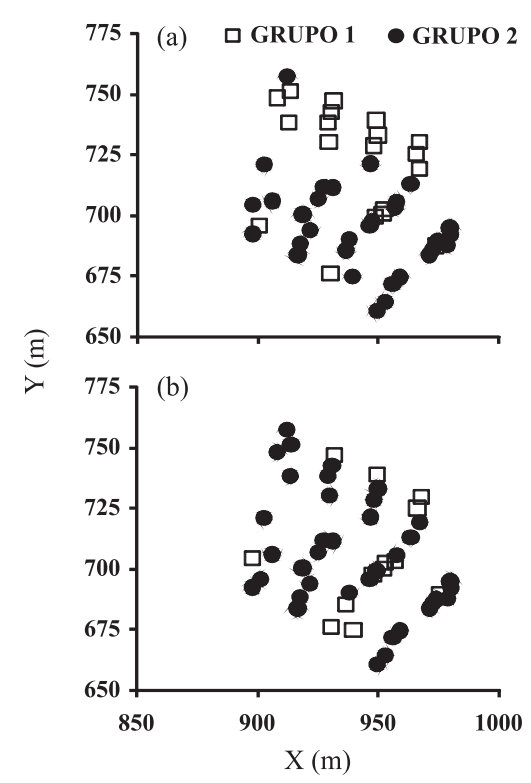

Figura 5. Distribuição dos pontos amostrais segundo a análise de agrupamento para as variáveis reais (a) e os cinco componentes principais (b).

do solo em relação à análise com os 22 dados originais. No caso em questão, como a maior parte da variabilidade está contida no primeiro $(42,00 \%)$ e no segundo componente (13,51\%), a análise fica ainda mais simples.

A análise geoestatística foi realizada utilizando-se os valores do primeiro e do segundo componente principal, ajustando-se aos semivariogramas experimentais o modelo gaussiano e o esférico, respectivamente (Figura 6).

Observou-se a dependência espacial, com aumento gradual da semivariância até determinada distância, em que o valor se estabilizou (patamar) com alcance de 40,7 e 15,4 m para o primeiro (acidez do solo) e o 

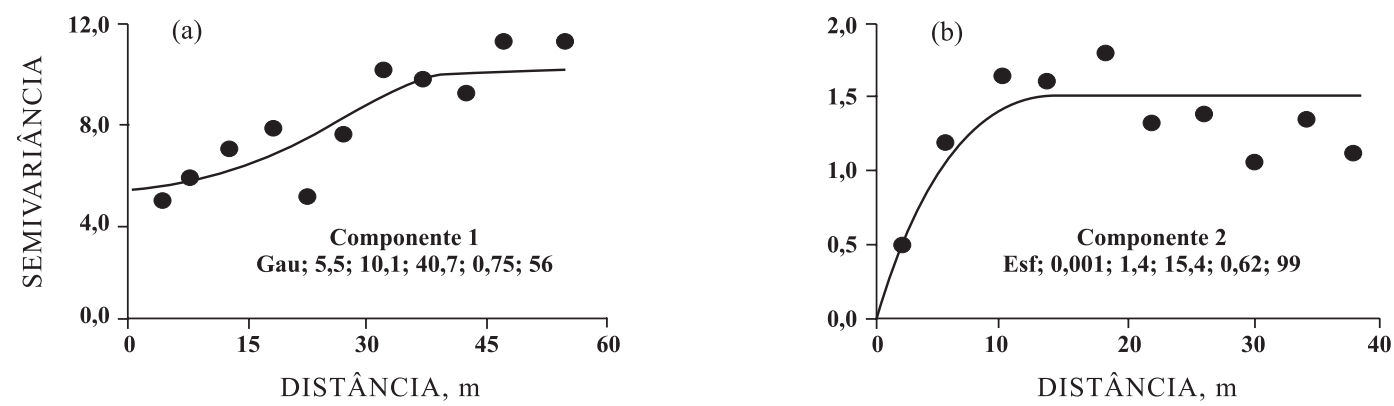

Figura 6. Modelos e parâmetros $\left(\mathrm{C}_{0} ; \mathrm{C}_{0}+\mathrm{C} ; \mathrm{a} ; \mathrm{R}^{2}\right.$; IDE) dos semivariogramas ajustados ao primeiro e ao segundo componente principal: a) gaussiano; b) esférico.

segundo componente principal (teor de matéria orgânica), respectivamente. Nesses casos, os pontos localizados numa área de raio menor ou igual ao alcance são mais similares e estão dependentes espacialmente entre si, podendo ser usados para estimar valores para locais não amostrados. Os valores de alcance podem influenciar a qualidade das estimativas, uma vez que eles determinam o número de valores usados na interpolação; assim, estimativas feitas com interpolação por krigagem ordinária utilizando valores de alcances maiores tendem a ser mais confiáveis, apresentando mapas que representam melhor a realidade (Corá et al., 2004). O índice de dependência espacial (IDE) mostrou moderada dependência para o primeiro componente e forte para o segundo, de acordo com classificação proposta por Cambardella et al. (1994).

Após definição dos modelos e parâmetros dos semivariogramas, os dados foram interpolados por meio da krigagem ordinária para mapear a acidez e o teor de matéria orgânica do solo, segundo os componentes selecionados (Figura 7).

Os mapas da figura 7 refletem, conjuntamente, 55,51 \% da variabilidade contida na área. Observouse que a acidez do solo (Figura 6a) é maior nas menores altitudes do terreno, porém as áreas com maior teor de Al trocável localizaram-se no topo da encosta, indicando grande quantidade de cargas $\mathrm{pH}$ dependentes no solo em estudo - fato comum nos Latossolos (Pierangeli et al., 2005). Oliveira (2007) e Silva et al. (2008) também encontraram maior disponibilidade de Al trocável nas porções superiores das áreas.

Por sua vez, a fração orgânica do solo apresentou distribuição mais complexa, com regiões bem definidas, sendo maior na parte inferior e na extremidade da parte superior esquerda, com os menores valores concentrados na região central da área (Figura 7b). Uma explicação pode ser o indício de menor acidez do solo na região central da área, o que acelera a decomposição da matéria orgânica e pode ter, também, reduzido os valores de CTC, que seguem o mesmo comportamento da MO.
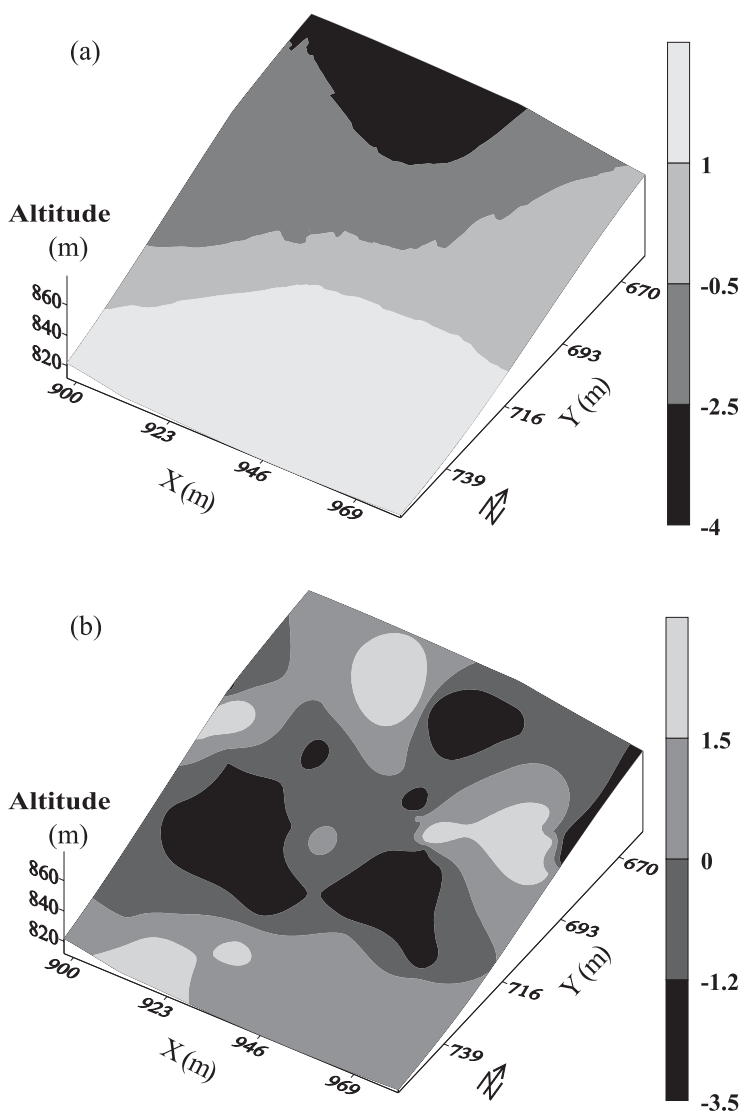

Figura 7. Mapa do primeiro (fator acidez do solo) (a) e do segundo (fator teor de matéria orgânica do solo) (b) componente principal. Os valores incluídos no eixo $\mathrm{Z}$ correspondem à altitude do terreno e os valores apresentados na legenda correspondem aos escores dos componentes selecionados.

\section{CONCLUSÕES}

1. O método de análise multivariada baseado nos componentes principais forneceu componentes interpretáveis, sendo o primeiro correlacionado com a acidez e o segundo com a matéria orgânica do solo. 
2. As técnicas de análise multivariada, em associação com a geoestatística, facilitaram a avaliação da variabilidade do solo; os componentes principais 1 e 2 apresentaram dependência espacial moderada, com maior continuidade espacial observada no componente 1, o que permitiu melhor caracterização da acidez do solo.

3. O solo em estudo apresentou, ao longo da encosta, disponibilidade reduzida de nutrientes, considerável acidez, menores teores de matéria orgânica na porção central da área e valores elevados de $\mathrm{Al}$ trocável na sua porção superior.

\section{AGRADECIMENTOS}

À CAPES, pela concessão de bolsa de mestrado.

\section{LITERATURA CITADA}

BUENO, B.F. Aplicação de técnicas multivariadas em mapeamento e interpretação de parâmetros do solo. Campinas, Universidade Estadual de Campinas, 2001. 84p. (Tese de Mestrado)

CAMBARDELLA, C.A.; MOORMAN, T.B.; NOVAK, J.M.; PARKIN, T.B.; KARLEN, D.L.; TURCO, R.F. \& KONOPKA, A.E. Field-scale variability of soil properties in Central Iowa soils. Soil Sci. Soc. Am. J. 58:1501-1511, 1994.

CORÁ, J.E.; ARAUJO, A.V.; PEREIRA, G.T. \& BERALDO, J.M.G. Variabilidade espacial de atributos do solo para adoção do sistema de agricultura de precisão na cultura de cana-de-açúcar. R. Bras. Ci. Solo, 28:1013-1021, 2004.

EMPRESA BRASILEIRA DE PESQUISA AGROPECUÁRIA . EMBRAPA. Centro Nacional de Pesquisa de Solos. Sistema brasileiro de classificação de solos. Rio de Janeiro, 2006. 306p.

EMPRESA BRASILEIRA DE PESQUISA AGROPECUÁRIA EMBRAPA. Centro Nacional de Pesquisa de Solos. Manual de métodos de análise de solo. 2.ed. Rio de Janeiro, 1997.

GANDAH, M.; STEIN, A.; BROUWER, J. \& BOUMA, J. Dynamics of spatial variability of millet growth and yields at three sites in Niger, West Africa and implications for precision agriculture research. Agric. Systems, 63:123$140,2000$.

GUIMARÃES, E.C. Variabilidade espacial de atributos de um Latossolo Vermelho-Escuro textura argilosa da região do cerrado, submetido ao plantio direto e ao plantio convencional. Campinas, Universidade Estadual de Campinas, 2000. 85p. (Tese de Doutorado)

GURGEL, H.C. Variabilidade espacial e temporal do NDVI sobre o Brasil e suas conexões com o clima. São José dos Campos, Instituto Nacional de Pesquisas Espaciais, 2003. (Tese de Mestrado)

KER, J.C. Latossolos do Brasil: Uma revisão. Geonomos, 5:1740, 1997.

LIPS, J.M. \& DUIVENVOORDEN, J.F. Regional patterns of well drained upland soil differentiation in the middle Caquetá basin of Colombian Amazonia. Geoderma, 72:219-257, 1996.
MANLAY, R.J.; CADET, P.; THIOULOUSE, J. \& CHOTTE, J. Relationships between abiotic and biotic soil properties during fallow periods in the sudanian zone of Senegal. Appl. Soil Ecol., 14:89-101, 2000.

MINGOTI, S.A. Análise de dados através de métodos de estatística multivariada. Belo Horizonte, Universidade Federal de Minas Gerais, 2007. 295p.

MOTTA, P.E.F.; CURI, N.; OLIVEIRA-FILHO, A.T. \& GOMES, J.B.V. Ocorrência de macaúba em Minas Gerais: Relação com atributos climáticos, pedológicos e vegetacionais. Pesq. Agropec. Bras., 37:1023-1031, 2002.

OLIVEIRA, R.B. Mapeamento e correlação de atributos do solo e de plantas de café conilon para fins de agricultura de precisão. Alegre, Universidade Federal do Espírito Santo, 2007.129p. (Tese de Mestrado)

PIERANGELI, M.A.P.; GUILHERME, L.R.G.; CURI, N.; SILVA, M.L.N.; LIMA, J.M. \& COSTA, E.T.S. Efeito do $\mathrm{pH}$ na adsorção e dessorção de cádmio em Latossolos brasileiros. R. Bras. Ci. Solo, 29:523-532, 2005.

RIBEIRO, A.C.; GUIMARÃES, P.T.G. \& ALVAREZ V., H.V., eds. Recomendações para uso de corretivos e fertilizantes em Minas Gerais. $5^{a}$ Aproximação. Viçosa, MG, Comissão de Fertilidade do Solo do Estado de Minas Gerais CFSEMG, 1999. 359p.

ROBERTSON, G.P. GS+: Geoestatistics for the environmental sciencies . GS+ User.s Guide. Plainwell, Gamma Desing Software, 1998. 152p.

SILVA, F.M.; SOUZA, Z.M.; FIGUEIREDO, C.A.P.; VIEIRA, L.H.S. \& OLIVEIRA, E. Variabilidade espacial de atributos químicos e produtividade da cultura do café em duas safras agrícolas. Ci. Agrotec., 32:231-241, 2008.

SILVA, P.C.M. \& CHAVES, L.H.G. Avaliação e variabilidade espacial de fósforo, potássio e matéria orgânica em alissolos. R. Bras. Eng. Agríc. Amb., 5:431-436, 2001.

SPLECHTNA, B.E. \& KLINKA, K. Quantitative characterization of nutrient regimes of high-elevation forest soils in the southern coastal region of British Columbia, Canada. Geoderma, 102:153-174, 2001.

STATSOFT. Statistica for Windows. Release 5.1 (Computer program manual). Yulsa, StatSoft Inc., 1997. Não paginado.

VIEIRA, S.R.; HATFIELD, J.L.; NIELSEN, D.R. \& BIGGAR, J.W. Geostatistical theory and application to variability of some agronomical properties. Hilgardia, 51:1-75, 1983.

VIEIRA, S.R. Geoestatística em estudos de variabilidade espacial do solo. In: NOVAIS, R.F.; ALVAREZ V., V.H. \& SCHAEFER, C.E.G.R. Tópicos em ciência do solo. Viçosa, MG, Sociedade Brasileira de Ciência do Solo, 2000. v.1. p.1-54.

WARRICK, A.W. \& NIELSEN, D.R. Spatial variability of soil physical properties in the field. In: HILLEL, D., ed. Applications of soil physics. New York, Academic Press, 1980. p.319-344.

ZWICK, W.R. \& VELICER, W.F. Factors influencing four rules for determining the number of components to retain. Psychol. B., 99:432-442, 1986. 